\title{
Chagas: Arte y Ciencia. Notas de un recorrido en construcción para abordar un problema complejo
}

Mariana Sanmartino

Grupo de Didáctica de las Ciencias (IFLYSIB, CONICET- UNLP), La Plata, Argentina

\section{Resumen}

$\mathrm{Si}$ bien el Chagas ha sido tradicionalmente concebido como un tema de estricto abordaje biomédico, debido a la multiplicidad de factores que lo caracterizan y a la diversidad de actores implicados, es necesario considerarlo como un problema verdaderamente complejo. Esto requiere realizar una lectura crítica de la problemática que permita desarrollar respuestas efectivas, sostenidas en el tiempo y acordes a las realidades socioculturales de los distintos contextos donde el Chagas existe. Compartiremos en este espacio un camino que estamos transitando desde hace un tiempo a través de ideas y experiencias plasmadas en actividades, materiales didácticos y recursos que pretenden complejizar la mirada sobre esta temática ambiental y de salud, aportando dimensiones usualmente no consideradas. Arte, ciencia y saberes populares se ponen en juego y se integran dinámicamente en una serie de propuestas que venimos desarrollando para invitarnos/invitarlos a mirar y sentir más allá de las dicotomías y de los abordajes tradicionales. Articulamos estas páginas a partir del texto del video "CHAGAS. Reconocer miradas, sumar voces, acortar distancias" (Argentina, 2010), un material audiovisual que refleja un posicionamiento particular, en el cual el Chagas es reinterpretado desde lugares no convencionales.

Palabras clave: Chagas, ciencias, arte, miradas, complejidad

Correspondencia: Mariana Sanmartino

Grupo de Didáctica de las Ciencias (IFLYSIB, CONICET- UNLP). Calle 55 Nro.910, 1900 La Plata, Provincia de Buenos Aires, Argentina Tel: +54 - 221- 4226042

Email: mariana.sanmartino@gmail.com
Chagas: Art and Science.

Notes from a course in construction to address a complex problem

\begin{abstract}
Although Chagas disease has been traditionally conceived as a matter of strict biomedical approach, due to the multiplicity of factors that characterize the subject and the diversity of actors involved, it is necessary to consider it a truly complex problem. This requires making a critical reading of the problem that allow us to develop effective, sustained over time and consistent actions with the sociocultural realities of the different contexts where Chagas exists. In this space we share the path that we are walking through ideas and experiences reflected in activities, materials and resources intended to make more complex the look on this environmental and health issue, adding dimensions usually not considered. Art, science and popular knowledge are at stake and are integrated dynamically into a series of proposals that we are developing to invite to look and feel beyond the dichotomies and traditional approaches. We articulate these pages from the text of the video "CHAGAS. Recognizing views, amassing voices, shortening distances "(Argentine, 2010), an audiovisual material that reflects a particular position in which Chagas disease is reinterpreted from unconventional places.
\end{abstract}

Keywords: Chagas, science, art, looks, complexity 
DOI: 10.5031/v1i2.RIA10169

\section{INTRODUCCIÓN}

Desde un punto de vista biológico o médico, el Chagas (también conocido como Tripanosomiasis americana) puede ser definido como una enfermedad crónica, causada por un parásito cuyo nombre científico es Trypanosoma cruzi. Este parásito es transmitido al ser humano $y$ otros animales a través de unos insectos que se alimentan de sangre. Estos insectos están presentes a lo largo de casi todo el continente americano $y$, según la región se conocen con diferentes nombres, como vinchuca, barbeiro, chinche picuda, chipo, pito, chirimacha, entre otros. Estas chinches dejan sus excrementos sobre la piel de sus víctimas en el momento de alimentarse, $y$ es precisamente en su materia fecal donde se encuentran los parásitos que, luego de ingresar al torrente sanguíneo, provocarán la infección. Otras vías de transmisión del parásito que causa el Chagas, menos frecuentes, son la transfusión de sangre o el transplante de órganos de donantes infectados, la transmisión de madres infectadas a sus bebés durante el embarazo o el parto y el consumo de bebidas y alimentos contaminados con los excrementos de los insectos vectores. El Chagas no se transmite a través de relaciones sexuales, besos, abrazos, ni por compartir una bebida, ni al dar la mano.

Respecto a la evolución de la enfermedad, después de 10 días de haber adquirido el parásito, la persona infectada atraviesa un período generalmente asintomático o que presenta síntomas generales como dolor de cabeza, fiebre prolongada y malestar general. Luego de dos ó tres meses, comienza una etapa también sin sintomas donde los análisis de laboratorio dan positivos, pero no se observan alteraciones orgánicas atribuibles al Chagas. De cada 10 personas que tienen Chagas, aproximadamente 3 desarrollan la enfermedad 20 ó 30 años después de haber sido infectadas evolucionando hacia una etapa crónica en la que presentarán lesiones de diversa complejidad principalmente en el corazón, pero también en el aparato digestivo o el sistema nervioso.

Parece existir un consenso generalizado en el ámbito científico y sanitario en considerar al
Chagas como uno de los problemas de salud pública más graves de América Latina. Sin embargo, no se cuenta con cifras que representen de manera confiable la magnitud real de la endemia, estimando que en la región existen -al menos- 10 millones de personas infectadas. Las estadísticas imprecisas son un claro resultado del sinnúmero de elementos que entran en juego y constituyen el entramado de esta problemática.

Tradicionalmente el Chagas ha sido considerado como un tema de estricto abordaje biológico y médico, lo que ha marcado un abismo entre la cantidad de conocimiento acumulado acerca de los aspectos biomédicos y aquel conocimiento referido a los factores sociales que caracterizan a este complejo problema. Cuando ampliamos la mirada más allá de esta visión tradicional nos encontramos con más preguntas que respuestas. Preguntas que hacen al contexto de la realidad socioeconómica de las poblaciones rurales, semirurales y de las periferias urbanas de América Latina.

El escenario general se hace más complejo aún cuando constatamos que en las últimas décadas, debido a los crecientes movimientos migratorios, el Chagas dejó de ser un problema exclusivamente rural y también dejó de ser una realidad exclusivamente latinoamericana. ${ }^{\mathrm{i}}$

\section{CIENCIA Y CHAGAS, CLARO. PERO, ¿ARTE Y CHAGAS?}

Cuando se habla de Chagas, en muchos ámbitos puede resultar casi natural la relación que tiene el "sector científico" con el tema. Además, es indiscutible el rol que cumplen muchas de las Ciencias Médicas, las Naturales y también las Exactas en la búsqueda de soluciones y conocimientos esclarecedores en torno a esta problemática. En este sentido, "la ciencia" ha avanzado y mucho en cuanto al

\footnotetext{
' Texto extraído del video "CHAGAS. Reconocer miradas, sumar voces, acortar distancias" (Sanmartino y colaboradores, 2010). Disponible en: $\quad$ http://vimeo.com/20341666 http://www.youtube.com/watch?v=rq8J8E4vPiw\& feature=related
} 
abordaje de la enfermedad, sin embargo quedan voces sin ser escuchadas, restan múltiples miradas a tener en cuenta y existe un sinnúmero de diálogos pendientes. Todo esto se traduce en el hecho de que, a más de 100 años de las primeras publicaciones científicas referidas al tema, éste siga siendo un gravísimo problema para millones de personas, cuya solución definitiva es aún difícil vislumbrar.

Por otra parte, cuando pensamos en el vínculo entre "otras ciencias" y Chagas, el tema de los roles y la pertinencia se vuelve lamentablemente- más discutible para algunos. Conocemos el parásito, sabemos de los hábitos del insecto; pero apenas entendemos, o sólo tenemos hipótesis generales, sobre el comportamiento del individuo que la padece, decía en 1990 el sociólogo venezolano Roberto Briceño-León ${ }^{1}$. Esta apreciación sigue vigente, en parte porque aún no se ha dado lugar de manera sistemática al aporte de las Ciencias Sociales y en parte también porque es necesario recordar que los verdaderos protagonistas de esta historia son las personas que padecen de alguna manera las consecuencias de este flagelo. Recordar esto implica considerar a esas personas como sujetos activos de las decisiones y las respuestas y no como destinatarios pasivos de acciones, muchas veces pensadas a cientos de kilómetros (geográficos o culturales) de donde el Chagas existe.

Es necesario reconocer que en realidad, cuando se habla de Chagas se dice mucho más de lo que se cree. El Chagas no es sólo una enfermedad, es un problema sumamente complejo, y considerarlo de esta manera implica abordar cuestiones como la pobreza, la discriminación, la toma de decisiones, además de aspectos culturales, educativos, ambientales y sociales. En ese sentido, romper con enfoques clásicos significa también romper con soluciones lineales y simplistas. No tenemos más remedio que ser imaginativos, flexibles y desprejuiciados, dijo Carlos Moreli en $1999^{2}$, reflexionando sobre

\footnotetext{
ii En el año 1999, el Dr. Carlos Morel era el director del Programa Especial para la Investigación y la Educación en Enfermedades
}

los pasos a seguir en la investigación sobre los diferentes aspectos del Chagas. Sería deseable también que tanto quienes abordamos esta problemática -desde cualquier sector y disciplinacomo quienes lo perciben como un tema lejano en el tiempo y/o en el espacio, nos sintiéramos interpelados por este llamado a mirar el tema de otra manera, respetando todas las voces y miradas. Para esto, no tenemos más remedio que ser, además, humildes y autocríticos.

En esta necesidad de sumar saberes y sentires, estamos hoy participando de un nuevo desafío, el de involucrar y sentar en la misma mesa de debate a representantes de otros "universos" diferentes del científico o médico asistencial. Si planteamos entonces, por ejemplo, la relación Arte y Chagas, el vínculo ya no parece tan natural para la gran mayoría de los interlocutores. Sin embargo, el camino recorrido (y por recorrer) a través de propuestas desarrolladas y ejemplos mencionados en el siguiente apartado demuestran no sólo que sí es posible la mirada del Arte sobre el Chagas, sino que es necesaria y recomendable. El Arte convoca e interpela a las personas desde lugares a los cuales la Ciencia no llegaría jamás, aportando sensibilidad y profundidad en la interpretación y análisis de temas como éste.

\section{LOS PASOS DADOS}

La vinculación del Arte, con la Ciencia y el Chagas, es algo en lo que venimos trabajando desde hace un tiempo. Los caminos transitados nos han permitido descubrir producciones sobre el Chagas a veces inimaginadas, creaciones y expresiones de diversa naturaleza que están ahí, diciendo algo desde lugares, miradas y voces normalmente no considerados a la hora de pensar en esta problemática tan particular y compleja.

A partir de lo expuesto, el recorrido aquí presentado brevemente se enmarca también en la necesidad de cuestionar el desarrollo de recursos y estrategias de comunicación y educación donde los destinatarios sean

Tropicales (TDR) de la Organización Mundial de la Salud. 
DOI: $10.5031 / \mathrm{v} 1 \mathrm{i} 2 . \mathrm{RIA} 10169$

considerados como receptores pasivos. Buscamos más bien propiciar espacios de investigación, debate y aprendizaje donde todos los actores involucrados participen activamente en las diferentes instancias y donde no sea la voz médica o "científica" la única autorizada a hablar de la problemática en cuestión.

Para facilitar su exposición, a modo de enumeración en orden cronológico destacamos algunos de los principales antecedentes que dan cuenta del recorrido llevado adelante hasta el momento:

- El taller "Algunos cuadros, un reggae y un poema... raras excusas para hablar de Chagas", dictado en "La Vidriera de los científicos del CONICET", en el marco del Espacio Joven de la $36^{a}$ Feria Internacional del
Libro de Buenos Aires en mayo de 2010. Actividad para alumnos de escuelas primarias y secundarias que consistía en presentar la problemática del Chagas de manera no convencional, con el objetivo de interpelar a los participantes y promover el intercambio de preguntas y reflexiones.

- El seminario-taller "La problemática del Chagas, ahora también urbana y global. Nuevas respuestas para nuevos escenarios" dictado junto con el pintor Néstor Favre-Mossier en el marco de la exposición de cuadros de su serie CHAGAS, durante la $20^{\mathrm{a}}$ Conferencia Mundial de Promoción de la Salud de la UIPES (Unión Internacional de Promoción de la Salud y Educación para la Salud) en Ginebra (Suiza) en julio de 2010 (Figura 1).

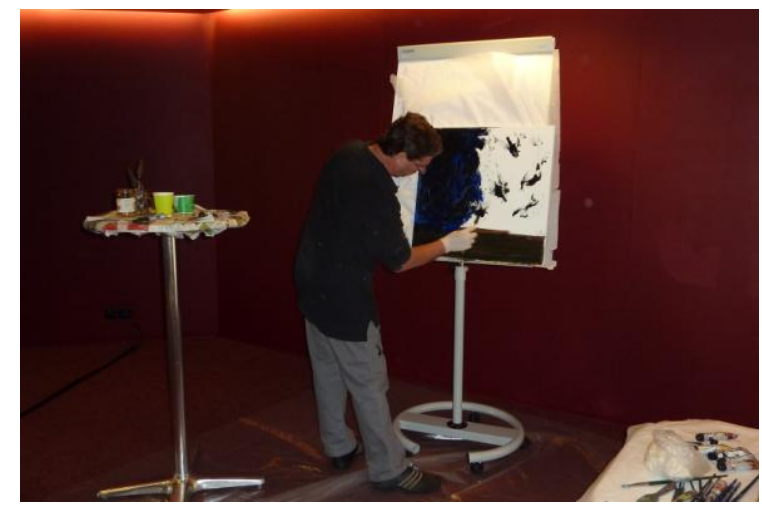

Figura 1: Imágenes tomadas durante el taller "La problemática del Chagas, ahora también urbana y global. Nuevas respuestas para nuevos escenarios" (Ginebra, julio de 2010).

- La realización de "CHAGAS. Reconocer miradas, sumar voces, acortar distancias", videoilustración de Sanmartino M, Costa JM, FavreMossier N, Mastropietro C y González R. Grupo de Didáctica de las Ciencias (IFLYSIB, CONICET-UNLP, La Plata) y El Birque Animaciones (Córdoba). 2010. En este material nos centraremos en el apartado siguiente dado que constituye al mismo tiempo una excelente síntesis y un ejemplo más que elocuente para el presente texto.

- "Arte, Ciencia y Chagas: miradas posibles, diálogos necesarios". Actividad cultural que contó con la participación (disertación y exposición de obras de la serie CHAGAS) del artista plástico argentino Néstor Favre-Mossier y la periodista y escritora chilena Ivonne Coñuecar. Organizada por la Red Chagas Entre Ríos y el Grupo de Didáctica de las Ciencias. Eventos declarados de Interés Provincial por la Honorable Cámara de Senadores de la Provincia de Entre Ríos y de Interés General por la Coordinación del Plan Nacer (Ministerio de Salud) Entre Ríos. Universidad Nacional del Litoral, Santa Fe (18 de noviembre) y Museo Provincial de Bellas Artes, Paraná (19 de noviembre), 2010. 
DOI: 10.5031/v1i2.RIA10169

- "Arte, Ciencia y Chagas: miradas posibles, diálogos necesarios. Memorias de un comienzo..." Sanmartino M, Ale ME (editoras). Edición Especial Coleccionable - El latir de los equipos, Nro.1. Plan Nacer Entre Ríos (Ministerio de Salud), ${ }^{\text {ii }} 2011$ (Figura 2). Textos de: Sanmartino M, Ale ME, Velázquez S, Saavedra S, Favre-Mossier N, Coñuecar I, Riobó P, Demonte $\mathrm{M}$, Olguín ME. Es una publicación que aborda la temática del Chagas desde una perspectiva que trata de rescatar, con algunos matices poco convencionales, diversas voces $y$ miradas. La revista es corolario de las dos actividades mencionadas en el punto anterior (realizadas durante noviembre de 2010 en las ciudades de Santa Fe y Paraná, Argentina); pero es resultado también de una búsqueda de saberes y experiencias que permitan pensar al Chagas trascendiendo el discurso estrictamente biomédico.

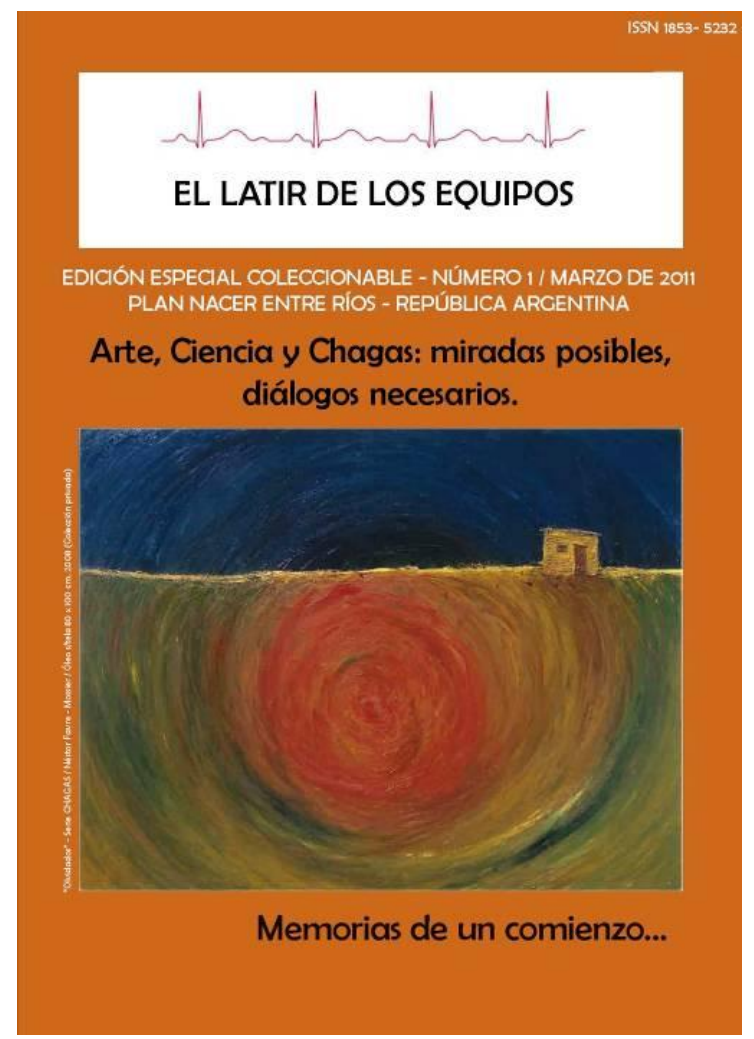

Figura 2: Portada de la revista.

\footnotetext{
iii La revista se puede consultar y descargar desde:

http://ripechagas.files.wordpress.com/2011/03/ellatir-de-los-equipos arte-ciencia-y-chagasweb2.pdf
}

- "Arte, Ciencia y Chagas: miradas posibles, diálogos necesarios, caminos transitados". Jornada cultural interdisciplinaria (para especialistas y público general) desarrollada en el Centro Científico Tecnológico del CONICET. La Plata. 31 de marzo de 2011.

- Semana del Chagas en el Museo de La Plata, FCNyM, UNLP. La iniciativa se enmarca en los trabajos de investigación, docencia y extensión de integrantes del Grupo de Didáctica de las Ciencias (IFLYSIB - CCT La Plata CONICET - UNLP). Para su organización se articuló con el Área Educativa del Museo de La Plata (Facultad de Ciencias Naturales y Museo, UNLP) y se contó con la participación de docentes e investigadores del Centro de Investigación Educativa de la Región I (CIE Región I, Dirección General de Cultura y Educación de la Provincia de Buenos Aires) y del Laboratorio de Triatominos (CEPAVE - CCT La Plata - CONICET - UNLP). Las actividades incluyeron una muestra de obras plásticas; la proyección de materiales audiovisuales innovadores; cuatro talleres con estudiantes de escuelas primarias y secundarias de la zona; cuatro charlas abiertas a cargo de especialistas; la presentación y distribución de materiales didácticos a docentes de escuelas; así como el desarrollo de actividades destinadas al público general. 9 al 15 de mayo de 2011 (Figura 3).

- "Arte, Ciencia y Chagas. Miradas posibles. ¿Diálogos necesarios? Homenaje al Dr. Eric Pairone". Jornada cultural interdisciplinaria desarrollada en el auditorio "Lic. Alicia Lucino de Bertoni" de la Fundación Espacios de Aprendizaje y Capacitación, Ciudad de Rafaela (Santa Fe, Argentina). 18 de agosto de 2011.

"CHAGAS. Reconocer miradas, sumar voces, acortar distancias..."

Tal como mencionamos anteriormente, consideramos al Chagas como un problema complejo y profundamente social que supera los límites de lo biomédico y por ello requiere aproximaciones de investigación, prevención y control que brinden respuestas integrales 


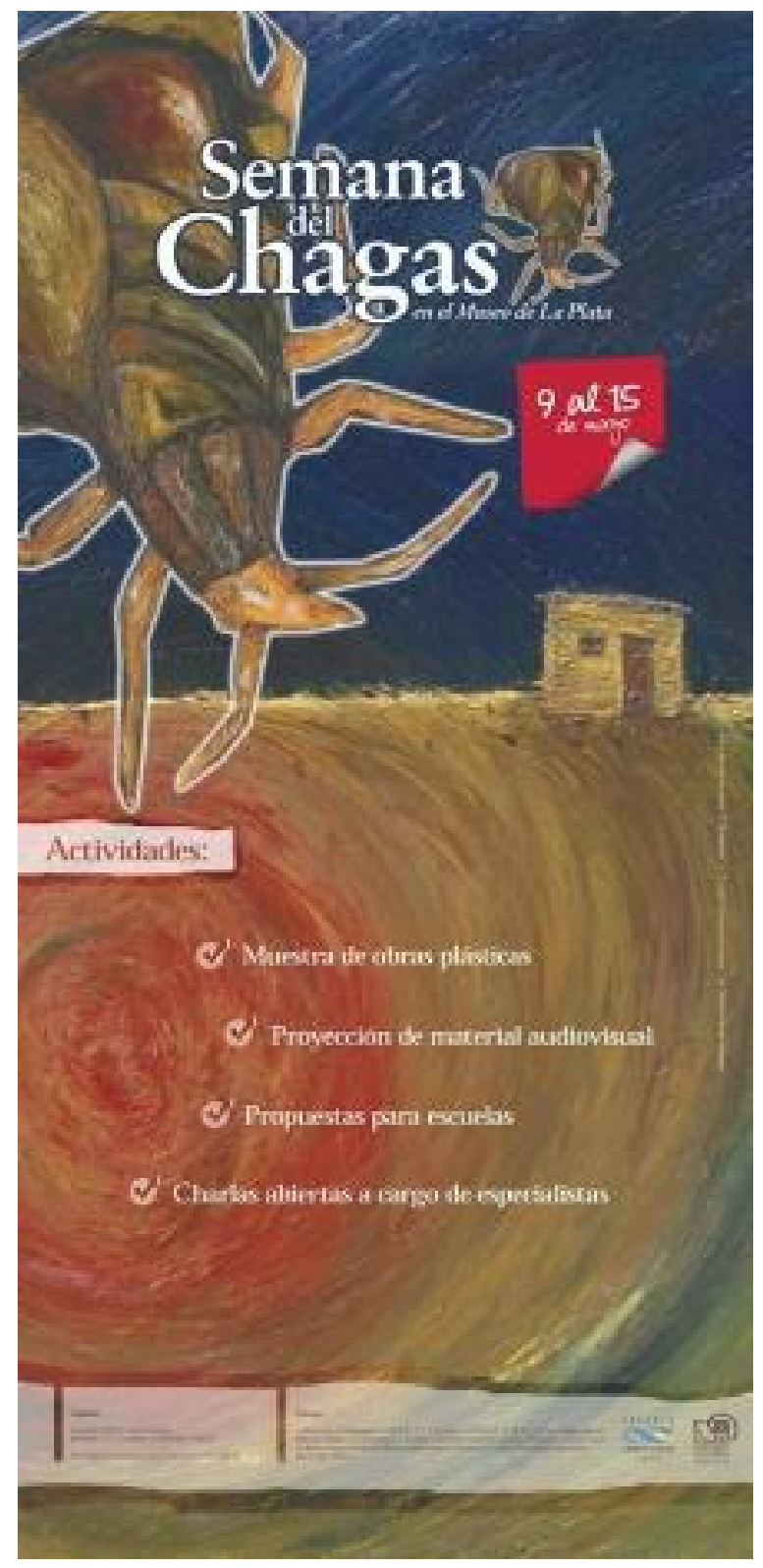

Figura 3: Banner de difusión de la Semana del Chagas en el Museo de La Plata (La Plata, mayo de 2011).

acordes a las características de los escenarios actuales (urbano y global, además de rural y latinoamericano). En este sentido, y para modificar la gran distancia que separa al "universo" de la producción científica, de las poblaciones afectadas, es necesario apuntar al desarrollo de estrategias y acciones que no estén orientadas sólo a evitar la enfermedad, sino a la promoción de la salud de las personas. A partir de estas consideraciones elaboramos "CHAGAS. Reconocer miradas, sumar voces, acortar distancias", un material audiovisual que refleja un posicionamiento particular, en el cual el Chagas es reinterpretado desde lugares no convencionales (el arte y la revalorización de las voces de los verdaderos protagonistas).

La propuesta aborda generalidades de la problemática desde una mirada integral, utilizando como soporte visual un video ilustración que muestra el proceso creativo de la realización de 5 imágenes en acrílico que ilustran los ejes temáticos del texto (Figura 4). En el proceso mismo de su realización se buscó dar lugar a la pluralidad de actores implicados con lo que el texto, la estructura, la imagen y el sonido (tanto de la música como de la voz) se conjugaron reflejando las particulares interpretaciones y sentires de los participantes para dar lugar a una obra colectiva con identidad propia.

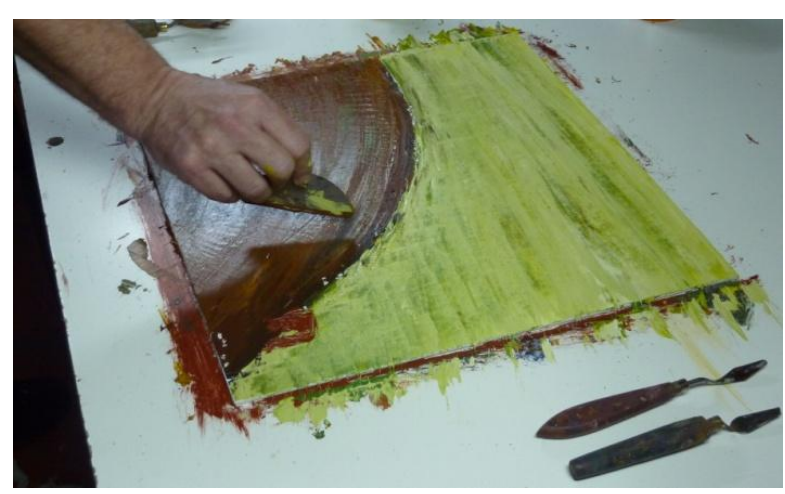

Figura 4: Imagen tomada durante la realización de "CHAGAS. Reconocer miradas, sumar voces, acortar distancias..." (Córdoba, septiembre de 2010).

La pieza no pretende brindar información exhaustiva del tema, ni abordar todos los aspectos que lo caracterizan sino más bien servir de "disparador" para pensar el Chagas de otra manera. El material puede ser utilizado en diferentes situaciones y contextos, por ejemplo: para plantear inicialmente el tema en una clase, como introducción (o cierre) de una disertación académica, para promover el debate en torno a alguna cuestión puntual, como disparador en un taller, etc.

Buscamos básicamente invitar a una reflexión colectiva y constructiva, apuntando a 
DOI: 10.5031/v1i2.RIA10169

sensibilizar e interpelar tanto al público especializado como al no especializado.

\section{Datos generales de la obra:}

Idea y texto: Mariana Sanmartino (CONICET, Grupo de Didáctica de las Ciencias, La Plata).

Realización: Juan Manuel Costa (EI Birque Animaciones, Córdoba)

Pinturas: Néstor Favre-Mossier (Artista plástico, Ciudad Autónoma de Buenos Aires)

Música original: Carlos Mastropietro (Compositor, La Plata)

Voz en off: Ramiro González (Escritor y Músico, La Rioja)

Revisión del texto: João Carlos Pinto Dias (Centro de Pesquisas René Rachou/CPqRR FIOCRUZ, Minas Gerais, Brasil)

Duración: 8:40

Año de realización: 2010

Lugar de realización: Córdoba y La Plata, Argentina

El video es de distribución libre y gratuita y está disponible en:

http://vimeo.com/20341666

http://www.youtube.com/watch?v=rq8J8E4vPiw\& feature=related

Registro: Esta obra está bajo una Licencia Reconocimiento-NoComercial-SinObraDerivada 3.0 Unported de Creative Commons.

\section{A MODO DE REFLEXIÓN}

Al inicio de este recorrido, fue que encontramos casi por casualidad una frase que sintetiza el objetivo final y el horizonte hacia el cual caminamos: ...es necesario analizar $y$ procurar modificar la gran distancia que separa a los laboratorios, los congresos y las publicaciones, de las poblaciones afectadas por el Chagas ${ }^{3}$.

Es a lo largo de este camino que, al intentar dar un cierre a cada uno de los pequeños grandes pasos dados, llega el momento del balance. Entonces miramos una vez más en perspectiva y reafirmamos que es en ese momento, al intentar imaginar y reflejar la compleja trama de elementos involucrados, cuando podemos empezar a entender verdaderamente de qué hablamos cuando hablamos del Chagas. Se hace fundamental entonces, entender que las respuestas que deben desarrollarse no pueden ser exclusivamente biológicas o médicas. Tanto en el entorno original como en los nuevos escenarios se necesitan respuestas que involucren de manera activa -y contemplando las particularidades de cada caso- a todos los actores implicados $y$ a todas las disciplinas $y$ campos de conocimiento que abordan el tema. Debemos entre todos y todas contemplar al Chagas desde una mirada integral que no esté orientada sólo a evitar la enfermedad, sino a la promoción de la salud de las poblaciones afectadas. (...) es necesario detenerse, hacer un balance del camino recorrido y encarar con una mirada integral los pasos a seguir. Detenerse también a contemplar y aprender de los caminos andados y desandados por quienes son los verdaderos protagonistas de esta historia: campesinas y campesinos, indígenas, inmigrantes en tierras lejanas, personas afectadas de una manera u otra por el Chagas y sus consecuencias (enfermedad, discriminación, falta de tratamiento, estigma). Mujeres, hombres, jóvenes, niños y niñas cuya voz aún no ha sido escuchada. Si realmente queremos modificar la gran distancia que separa a las poblaciones afectadas por este flagelo de los laboratorios, los congresos y las publicaciones, es necesario dar a sus palabras y sus saberes el peso y el valor de las palabras y saberes tradicionalmente autorizados para escribir y hablar de Chagas. ${ }^{\text {iv }}$

\section{AGRADECIMIENTOS}

Sería difícil nombrar aquí a todos los que hicieron y hacen posible la materialización de las ideas $y$ propuestas del recorrido aquí presentado. Sin embargo, no quiero dejar de mencionar que lo compartido en este espacio se sustenta en la colaboración desinteresada, la confianza y la voluntad de una gran cantidad de personas.

iv Texto extraído del video "CHAGAS. Reconocer miradas, sumar voces, acortar distancias" (Sanmartino y colaboradores, 2010). 
DOI: 10.5031/v1i2.RIA10169

\section{BIBLIOGRAFÍA}

1. Briceño-León R. La casa enferma. Sociología de la enfermedad de Chagas. Caracas: Fondo Editorial Acta Científica Venezolana, Consorcio de Ediciones Capriles; 1990. p. 29.

2. Morel C. Chagas Disease, from Discovery to Control - and Beyond: History, Myths and Lessons to Take Home. Mem. Inst. Oswaldo Cruz. 1999;94 (Suppl. 1):3-16.
3. Pinto Dias JC, Borges Dias R. Las viviendas y la lucha contra los vectores de la enfermedad de Chagas en el hombre, en el Estado de Minas Gerais, Brasil. Bol Oficina Sanit Panam. 1982;93:453-67. 